\title{
Future perspectives for physical education, sports and fitness.
}

DOI: $10.46981 /$ sfjhv2n2-007

Received in: April 1st, 2021

Accepted in: May 31th, 2021

\section{Estélio Henrique Martin Dantas}

Programa de Pós-graduação Stricto Sensu em Saúde e Ambiente - PSA, da Universidade Tiradentes UNIT, Aracaju, Brasil;

Programa de Pós-graduação Stricto Sensu em Enfermagem e Biociências - PPgEnfBio, da Universidade Federal do Estado do Rio de Janeiro - UNIRIO, Rio de Janeiro, Brasil; estelio.dantas@unirio.br

\section{Michael Douglas Celestino Bispo}

Programa de Pós-graduação Stricto Sensu em Saúde e Ambiente - PSA, da Universidade Tiradentes UNIT, Aracaju, Brasil; michaeldouglasc@hotmail.com

\section{Karollyni Bastos Andrade Dantas}

Programa de Pós-graduação Stricto Sensu em Saúde e Ambiente - PSA, da Universidade Tiradentes UNIT, Aracaju, Brasil;

karollynibastos@yahoo.com.br

\section{Cristiane Kelly Aquino dos Santos}

Programa de Pós-graduação Stricto Sensu em Saúde e Ambiente - PSA, da Universidade Tiradentes UNIT, Aracaju, Brasil;

Curso de Educação Física da Faculdade Fera, Arapiraca, Brasil;

ckellyakins@gmail.com

\section{Evelini Veras de Jesus}

Programa de Pós-graduação Stricto Sensu em Saúde e Ambiente - PSA, da Universidade Tiradentes UNIT, Aracaju, Brasil; evelimania@hotmail.com

\section{Lúcio Flávio Gomes Ribeiro da Costa}

Programa de Pós-graduação Stricto Sensu em Saúde e Ambiente - PSA, da Universidade Tiradentes UNIT, Aracaju, Brasil;

Curdo de Educação Física do Centro Universitário do Rio São Francisco, Paulo Afonso, Brasil; luciojudo@hotmail.com

\section{Pedro Alves de Figueiredo Neto}

Curso de Medicina da Universidade Tiradentes- UNIT, Aracaju, Brasil;

pedro17091997@gmail.com

\section{Dalva Teresinha de Souza Zardo Miranda}

Instituto Federal de Educação, Ciência e Tecnologia de Mato Grosso do Sul, Mato Grosso do Sul, Brasil. 


\section{RESUMO}

Nos dias atuais é cada vez mais corriqueiro o discurso sobre o ritmo acelerado dos acontecimentos e expansão das mudanças que vem ocorrendo da contemporaneidade social. O objetivo do presente artigo é discutir sobre as perspectivas futuras da Educação Física, esporte e fitness no cenário atual. A sociedade 5.0 é a resposta da humanidade para se alcançar os Objetivos de Desenvolvimento Sustentável do Milênio. As iniciativas aqui apresentadas refletem potencialmente a transformação do mundo em que vivemos, assegurando um futuro mais justo, sustentável e viável.

Palavras-Chave: Educação Física, Sociedade 5.0, Desenvolvimento Sustentável

\section{INTRODUCÃO.}

Os últimos anos vêm se caracterizando por um crescimento exponencial do conhecimento científico, da tecnologia da informação. Esses fatores estão acarretando profundas transformações da sociedade, no mercado de trabalho e nos meios de produção (Cunha, Cunha, Costa, \& Cunha, 2017).

Nos dias atuais é cada vez mais corriqueiro o discurso sobre o ritmo acelerado dos acontecimentos e expansão das mudanças que vem ocorrendo da contemporaneidade social, porém, um pensador americano, Alvin Tofler (1928 - 2016) percebeu esta tendência ainda na década de 60, quando difundiu conceitos inovadores no campo da política, economia e das tecnologias, lançando junto as suas ideias futuristas um novo ramo de atuação profissional baseado em seus pensamentos sobre planejamentos por cenários (Tofler, 1973).

Tofler (1973) afirmou que a sociedade humana evoluiu por meio de três grandes ondas de transformação: a primeira, ocorrida ainda no período neolítico, há cerca de dez mil anos antes de Cristo, foi a descoberta da agricultura e a domesticação de animais. Esse acontecimento nos transformou, de caçadores e coletores nômades, em agricultores e criadores estabelecidos.

Essa primeira onda no período Neolítico, foi ainda mais acentuada, cinco mil anos depois, pela invenção do arado que possibilitou o excedente de comida e, por conta disso, originando o que conhecemos hoje como cidade e depois a organização em civilizações (Santos, Veloso, \& Oliveira, 2017).

A segunda onda, ocorrida no início do século XVIII foi a Revolução Industrial decorrente da invenção da máquina a vapor. Até este evento, todas as realizações da civilização humana, as pirâmides de Gize, a Grande Muralha da China, Chichén Itzá, dependeram do dispêndio de força humana ou da tração animal para sua construção (Sachs, 2018).

A terceira onda, em 1980, foi a da telemática, uma combinação das telecomunicações com a informática, que transformaria radicalmente a organização do trabalho e difusão das mídias interativas, pela popularização das tecnologias da informação e avanços de alienação social, crises nas famílias, 
aumentos no uso de drogas ilícitas, terrorismos eletrônicos de países, bancos e empresas (Azevedo \& Santos, 2017).

Em função da crescente evolução tecnológica e da hiperconectividade, uma nova sociedade deverá surgir para adaptar-se às mudanças do contexto socioeconômico e das relações pessoais, ou smart society com a implementação da inteligência artificial (IA) em uma sociedade inteligente por uma gestão inteligente que garanta a qualidade e sustentabilidade das necessidades humanas e otimize as interações homem-máquina (Foresti et al., 2020).

O paradigma do novo sistema tecnológico da Sociedade 5.0 se baseia numa gestão centrada na essência da existência do homem, na integridade física e na interação com os recursos naturais (Sachsenmeier, 2016).

Em 2016, o Gabinete do Japão (que é o órgão diretivo do governo Japonês, constituído pelo Primeiro Ministro e pelos demais 19 titulares dos Ministérios) adotou a estratégia nacional de cinco anos $5^{\circ}$ Plano Básico de Ciência e Tecnologia formulada pelo Conselho de Ciência, Tecnologia e Inovação (CSTI) do governo japonês. Neste plano, foi proposta a Sociedade 5.0, como uma sociedade que o Japão deveria aspirar a ser implementada até 2021 (Man, 2018).

A sociedade superinteligente, a Sociedade $5.0 \mathrm{em}$ seu conceito abrangente de transformação completa do modo de vida através da inteligência artificial e robótica, se refere à ideia de um sistema econômico que além de sustentável é inclusivo e fortemente provido de tecnologias de sistemas ciberfísicos avançados, com o objetivo de transpor problemas sociais crônicos como o envelhecimento humano, o estresse do trânsito, a crescente polarização da sociedade, as restrições de fonte energética limpa, o acesso a baixo custo de tecnologias médicas e os problemas ambientais (UNESCO, 2015).

A Sociedade 5.0 decorre da evolução da sociedade através dos tempos, partindo de sociedade nômade, caçadora e extrativista (Sociedade 1.0); a sociedade agrícola e pastoril (Sociedade 2.0); a sociedade da indústria, (Sociedade 3.0); e a sociedade da informação (Sociedade 4.0). Será, portanto, uma sociedade posterior às evoluções sociais preconizadas por Tofler (Shiroishi, Uchiyama, \& Suzuki, 2018). 
Figura 1: A Evolução da Sociedade segundo o $5^{\circ}$ Plano Básico de Ciência e Tecnologia do Japão.

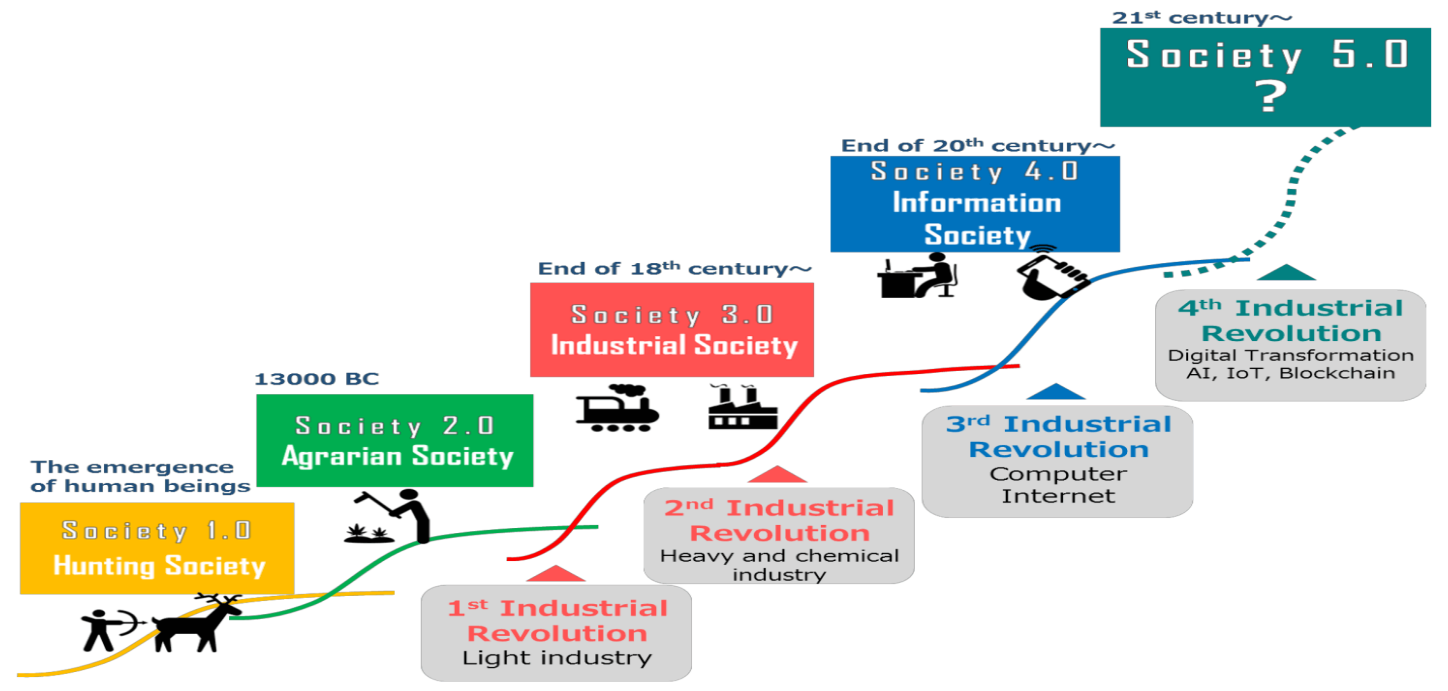

Fonte: Maeda, 2019

Das linhas de montagem desta nova sociedade são produzidos em tempo súbito inovações e artefatos personalizados. Essa tendência de fugir da linha de montagem padronizada para a fabricação de artigos personalizados de acordo com a vontade do cliente é a lógica da nova indústria. Para caracterizar esta inovação, foi criado em 2011 na Alemanha o termo Indústria 4.0, durante a Feira da Indústria de Hannover. Um estudo publicado em 2013, liderado por Siegfried Dais (Robert Bosch) e Kagermann (German Academy of Science and Engineering), teve como objetivo implementar um novo modelo de indústria para o Governo Federal Alemão, que trata da conexão entre máquinas, sistemas e ativos que possam promover ao sistema industrial um controle exato de cada etapa da cadeia de valor, para tornarem suas fábricas inteligentes, que foi divulgado na Feira anual de Hanover (Hnát, Herko, \& Gregor, 2018).

A nova fase na Revolução Industrial, a Indústria 4.0, também conhecida como Internet das Coisas (IoT) ou manufatura inteligente, é ditada pela interconectividade, automação, e aprendizado de máquina e dados em tempo real, interligando produção física e operações com tecnologia digital inteligente, redes de máquinas e dispositivos, computação em nuvem que centralize informações e com concentração para criar um ecossistema melhor conectado para empresas focadas na manufatura e no gerenciamento da cadeia de suprimentos, para promover uma evolução industrial sustentável para a existência do homem e suas dimensões social, ambiental e econômica, e mesmo operando de formas diferentes, as empresas e organizações, enfrentam comumente o desafio de necessitar de conectividade e acesso a insights em tempo real entre processos, parceiros, produtos e pessoas (Garcia-Muiña, González-Sánchez, \& Ferrari, 2018). 
A indústria 4.0 é fruto de uma evolução que se inicia na revolução industrial e que progride de acordo com o apresentado na Figura 2.

Figura 2: As quatro etapas da evolução da Indústria, segundo o Governo Alemão.

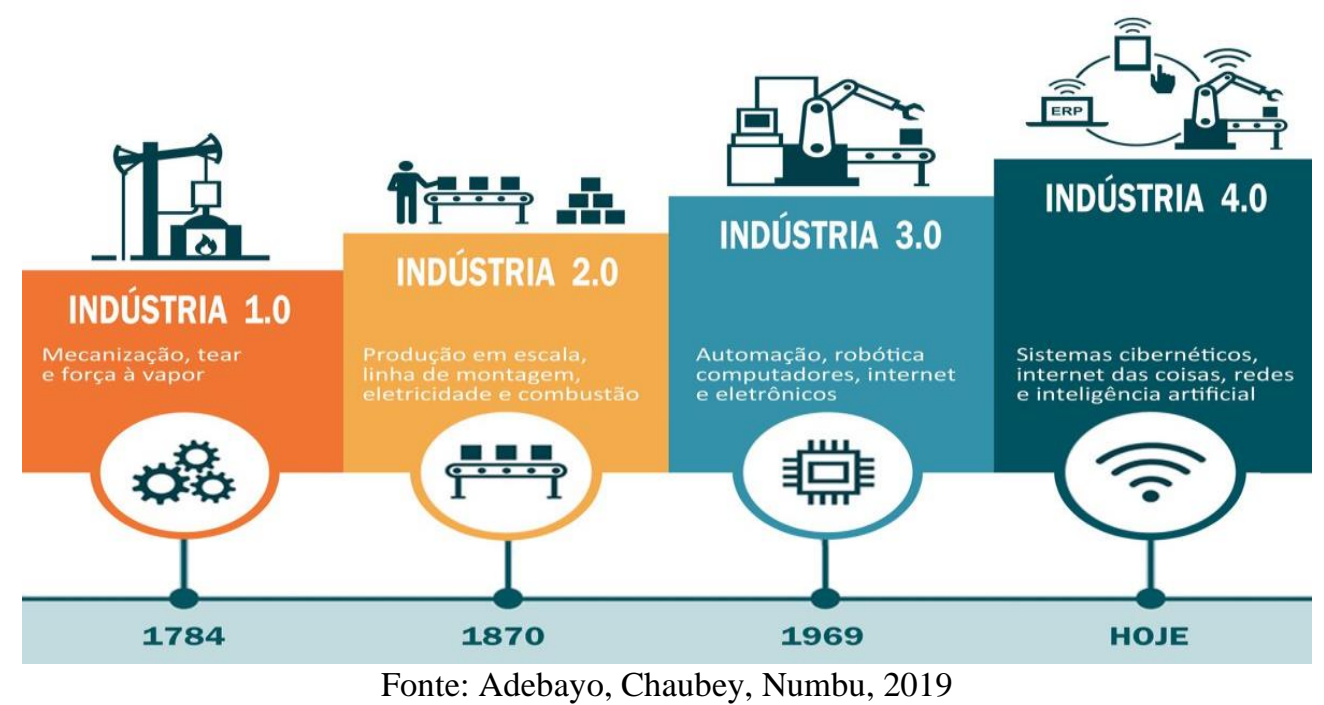

Nessa nova concepção de indústria, as atividades recorrentes passam a ser realizadas exclusivamente por máquinas, acarretando novas profissões que serão consolidadas no mercado de trabalho nos próximos cinco a dez anos.

Um estudo realizado pelo Serviço Nacional de Aprendizagem Industrial (SENAI) prevê advento de 30 novas ocupações nos níveis médio e superior, em oito áreas de grande relevância: automotivo; alimentos e bebidas; máquinas e ferramentas; petróleo e gás; têxtil e vestuário; química e petroquímica; tecnologias da informação e comunicação, e construção civil (Serviço Nacional da Industria - SENAI, 2018).

Esses novos profissionais, que deverão ser especialistas com competências atualizadas às necessidades futuras das empresas, devem adquirir qualificações para inovar, empreender, agilizar resoluções de problemas e capacidades de dominar as tecnologias digitais. A nova tendência são: as ferramentas tecnológicas que economizem trabalhos repetitivos; mais oportunidades de interpretação de dados; e tomada de decisões de forma mais avançada, a partir da inteligência artificial (Serviço Nacional da Industria - SENAI, 2018).

Outro fator condicionante da sociedade e do mercado de trabalho é a redução dos recursos da força de trabalho pelo envelhecimento populacional mundial, um fenômeno denominado "transição demográfica" que se caracteriza por uma mudança no comportamento em massa com a redução da fecundidade a níveis mínimos, redução da mortalidade e aumento da expectativa de vida, e acentuado aumento do grupo de idosos (Oliveira, 2019). 
No caso brasileiro, segundo a Divisão de População da ONU a transição demográfica entre 1950 e 2100, foi estimado que taxas brutas de mortalidade começaram a cair no final do século XIX e as taxas brutas de natalidade se mantiveram altas durante toda a história brasileira, tendo começado uma queda na década de 1960, entre 1950 a 1975 a idade média do brasileiro era próxima de 20 anos, o que significa dizer que metade da população tinha menos de 20 anos e que pessoas idosas acima de 60 anos representavam apenas 5\%, ou seja o Brasil era considerado um país com uma estrutura etária extremamente jovem (Alves, 2019).

Este cenário mudará por completo no final do século XXI, quando a idade mediana estará acima de 50 anos, mostrando que metade da população terá mais de 50 anos. Em termos percentuais o Brasil terá cerca de $40 \%$ de idosos de 60 anos, apresentando-se estruturalmente velho com decrescimento populacional, situação em que irão ser necessárias políticas econômicas e sociais adequadas às novas estruturas populacionais (Alves, 2019).

Figura 3: Mudanças da estrutura etária do Brasil: Idade mediana (em anos), 1950-2100.

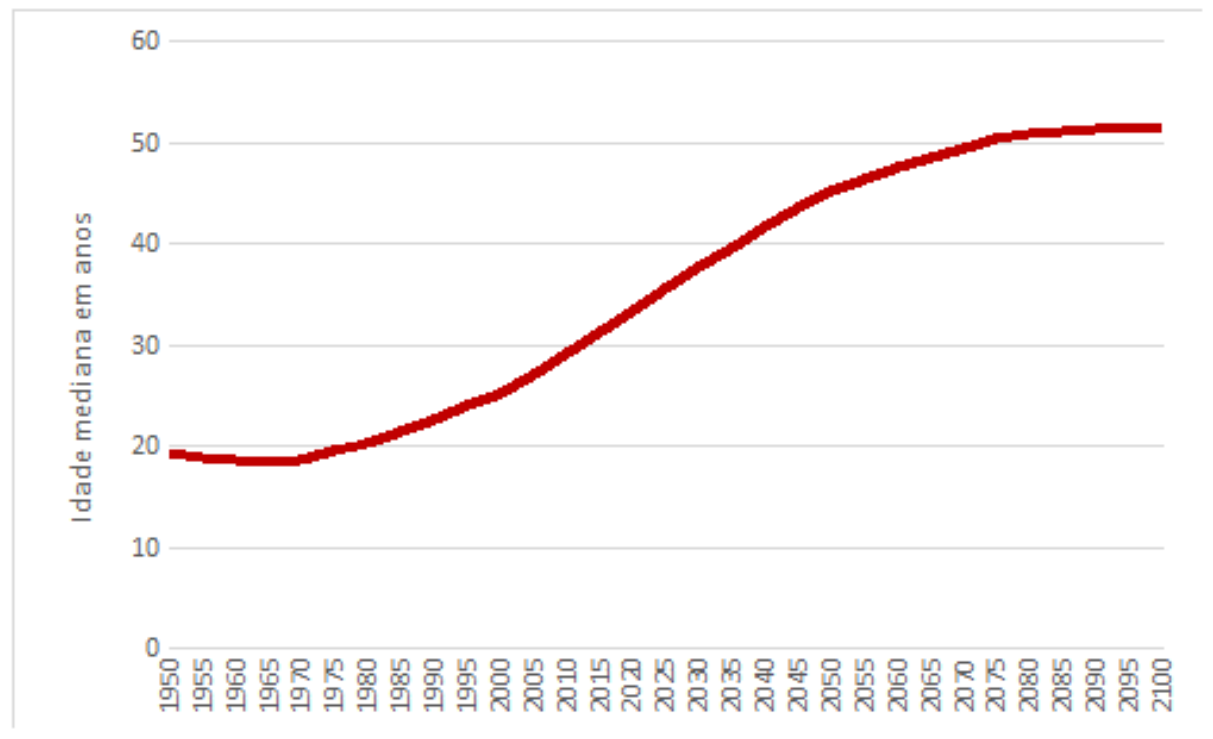

Fonte: UN/Pop Division: World Population Prospects, 2019.

Considerando esse quadro, o envelhecimento populacional está relacionado às mudanças que impactam a estrutura etária de uma população e consubstancialmente o aumento no percentual relativo das pessoas acima de uma idade determinada como o início da velhice, variando em diferentes sociedades, que considera além dos fatores biológicos, outros como econômicos, ambientais, científicos e culturais. Portanto cabe dizer que o fenômeno social da transição demográfica, epidemiológica da população e o envelhecimento é a representatividade do desfecho de ações orquestradas pela saúde pública que foi capaz de promover cuidados preventivos e curativos, modificando o cenário de mortalidade e aumentando a expectativa de vida (Borba-Pinheiro, Dantas, \& Figueiredo, 2016). 
Com o aumento da expectativa de vida da população mundial e, consequentemente, da população idosa, um estudo observou uma perda de habilidades cognitivas em idosos, que podem ser prevenidos e retardados através da prática de exercícios físicos além de promover benefícios psicossociais, comportamentais, na realização das atividades de vida diária, reduzir risco de quedas e fraturas, consequentemente menor número de hospitalizações (Doine; Martelli; Hunger, 2020).

Assim, prevenir não é somente promover uma condição física, sua completitude ocorre quando atende aspectos sociais, psicológicos, socioeconômicos, sociopolíticos, socioculturais e psicossociais (Vale, Pernambuco, \& Dantas, 2016).

Se no passado o Profissional de Educação Física era requerido, prioritariamente, para trabalhar com crianças e adultos, nos dias de hoje e no futuro, cada vez mais, sua clientela será de idosos. Para fazer frente a todas estas transformações, ocorridas em tão curto espaço de tempo e visando preservar os valores humanos, a Organização Mundial da Saúde lançou em setembro de 2015, a Agenda 2030, com os 17 Objetivos de Desenvolvimento Sustentável do Milênio (ODS). Esses objetivos se escalonam em três níveis: pessoal, ecológico e espiritual, conforme apresentado na Figura 4. (ONU, 2015)

Figura 4: Objetivos de Desenvolvimento Sustentável do Milênio (ODS)
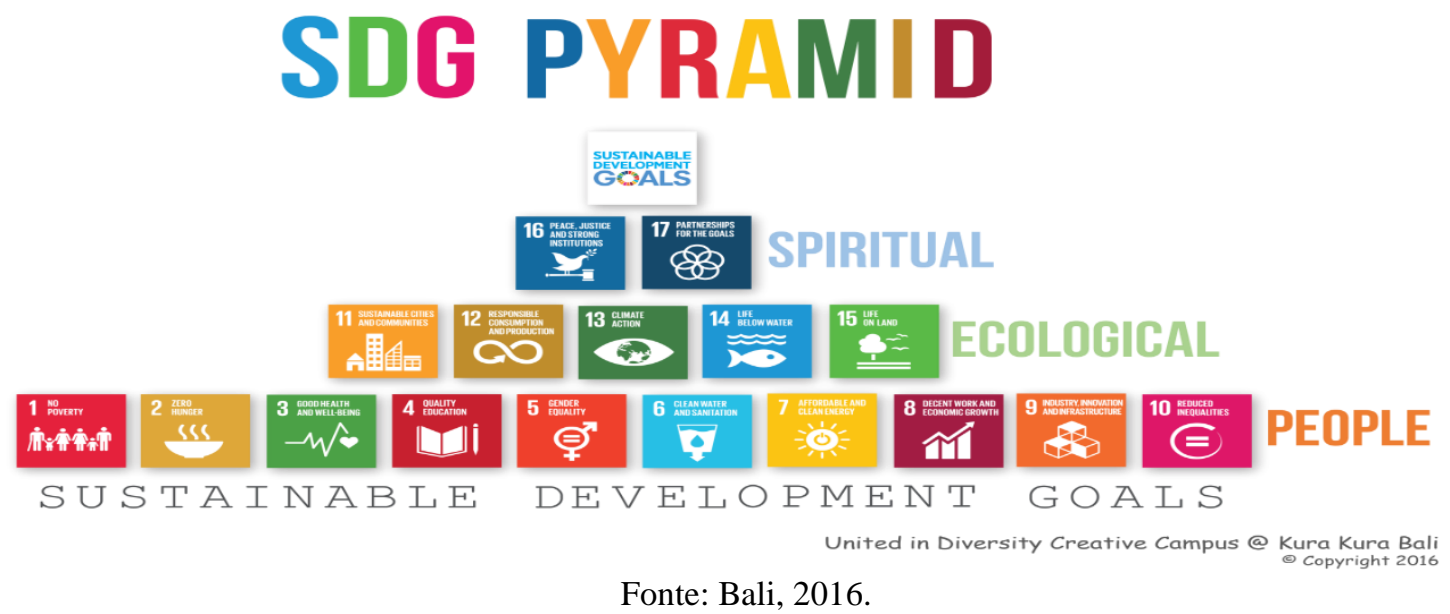

Essa agenda apresenta uma oportunidade histórica para melhorar os direitos e o bem-estar do ser humano de nosso planeta, em especial das pessoas em maior estado de vulnerabilidade como as crianças, os idosos, os enfermos e os mais desfavorecidos. Visando garantir um planeta saudável para as futuras gerações que estarão vivenciando as bruscas transformações sociais, tecnológicas e econômicas deste novo milênio.

No primeiro nível dos objetivos, o que trata das pessoas, as preocupações da ONU se centram em: pobreza, fome, saúde, educação, gênero, água, energia, trabalho, infraestrutura e desigualdade. O profissional de Educação Física além de se responsabilizar diretamente pela saúde e educação necessita 
harmonizar sua atuação laboral com os demais objetivos. A mesma preocupação de adequação na forma de agir profissionalmente deve ocorrer em relação aos objetivos ligados a Ecologia (sustentabilidade, desperdício, clima, diversidade e ambiente), bem como aos ligados à Espiritualidade (paz, justiça e participação).

\section{DESENVOLVIMENTO}

Neste início de milênio as transformações econômicas e sociais, impulsionadas pela tecnologia e pela inovação estão ocorrendo de forma exponencial, em uma escala nunca vista na história humana. Este fato está produzindo profundas modificações na saúde, no ensino e no mercado de trabalho.

Na Figura 5, apresenta-se a interação entre o mundo virtual e o físico, cada vez mais presente em nossa realidade. Os produtos e artigos físicos cada vez mais serão confeccionados de forma automatizada, a grande parte deles por meio de impressoras 3D, os serviços serão prestados por robôs. Todos os objetos e seres serão fontes de informação virtual, graças aos chips e sensores neles instalados. Por sua vez o Cyber Space será moldado por um gigantesco volume de informações (Big Data), analisáveis efetivamente apenas por meio de inteligências artificiais.

Figura 5: A relação entre a Realidade Virtual e o Mundo Físico

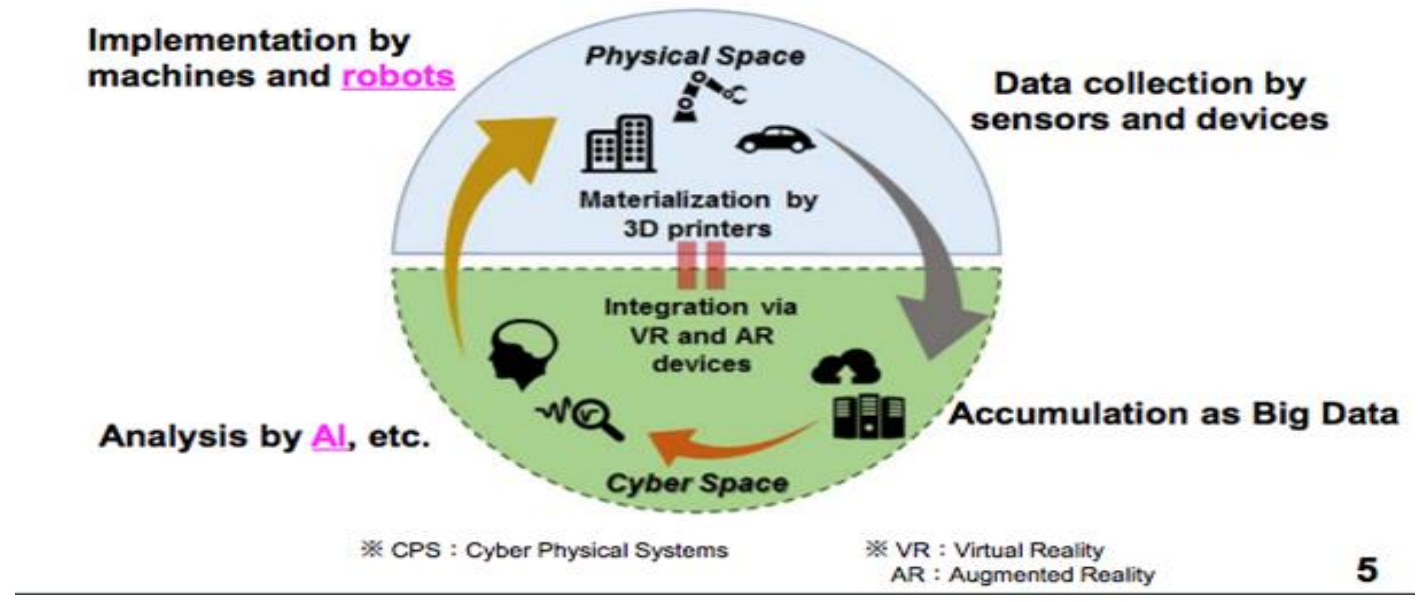

Fonte: Martynov, Shavaleeva, Zaytseva, 2019

O mercado de trabalho, como se pode esperar, sofrerá profundas transformações decorrentes destas novas tendências. Todas as pesquisas realizadas pela Singularity University e outras organizações profundamente envolvidas com inovação, apontam para a preservação e ampliação das profissões que não possam ser mais bem desempenhadas por máquinas ou por inteligência artificial, notadamente, as que dependam da criatividade, do cuidado e da atenção humana (Bersin, 2016).

Portanto, pode-se esperar um grande desenvolvimento nos próximos anos das profissões ligadas à: criação de conteúdos digitais; desenvolvimento de softwares; prestação de cuidados (enfermeiros, 
fisioterapeutas, terapeutas ocupacionais); e ao atendimento pessoal (baristas, enólogos, personal trainers, etc). (TIDD \& BESSANT, 2018).

O impacto da revolução tecnológica no mercado laboral de Educação Física, Esporte e Fitness passa, necessariamente, pela assimilação e ajuste às novas realidades e inovações apresentadas no presente ensaio.

O American College of Sports Medicine - ACSM, a cada ano, promove pesquisa mundial sobre as tendências do mercado de Fitness. A do ano de 2019 é apresentada na Figura 6 (Thompson, 2019).

Figura 6: Tendências do Mercado de Fitness em 2019

\begin{tabular}{|r|l|}
\hline 1 & Wearable technology \\
\hline 2 & Group training \\
\hline 3 & High-intensity interval training (HIIT) \\
\hline 4 & Fitness programs for older adults \\
\hline 5 & Bodyweight training \\
\hline 6 & Employing certified fitness professionals \\
\hline 7 & Yoga \\
\hline 8 & Personal training \\
\hline 9 & Functional fitness training \\
\hline 10 & Exercise is Medicine \\
\hline 11 & Health/wellness coaching \\
\hline 12 & Exercise for weight loss \\
\hline 13 & Mobile exercise apps \\
\hline 14 & Mobility/myofascial devices \\
\hline 15 & Worksite health promotion and workplace well-being programs \\
\hline 16 & Outcome measurements \\
\hline 17 & Outdoor activities \\
\hline 18 & Licensure for fitness professionals \\
\hline 19 & Small group personal training \\
\hline 20 & Postrehabilitation classes \\
\hline
\end{tabular}

Analisando-se o quadro da Figura 6, pode-se identificar algumas intenções que vêm se modificando ao longo dos anos, como: a progressiva utilização da tecnologia; a procura por serviços de qualidade; a necessidade de maior grau de atenção pessoal; e a promoção de modelos de exercícios como forma de obtenção da saúde.

Se no milênio passado os empregos na área de Educação Física se centravam na Educação Física Escolar, no esporte e no mercado de estética corporal, pode-se observar uma tendência de mobilidade para os segmentos de mercado com maior potencial de desenvolvimento fatorial, conforme apresentado do por Tidd \& Bessant (2018).

1) Criação de Conteúdos Digitais - notadamente no campo do esporte do alto rendimento e em todas as atuações correlatas: treinamento, promoção de competições, descoberta de talentos, orientação da vocação esportiva etc.;

2) Prestação de Cuidado - execução de serviços ligados à saúde, como por exemplo: o 
condicionamento físico de idosos, a Educação Física Hospitalar e o atendimento ambulatorial a grupos especiais e aos obesos e aos portadores de doenças crônicas (síndrome metabólica, hipertensão, diabetes, câncer, AIDS etc);

3) Atendimento Pessoal - materializado na prestação de serviços ajustados às necessidades individuais como: treinamento personalizado, personal training para grupos pequenos e o atendimento em estúdios.

Assim como na Medicina, na Nutrição e no Direito e em tantas outras profissões, algumas das tarefas do profissional de Educação Física serão extintas pela tecnologia (Maddox, Rumsfeld, \& Payne, 2018). A exemplo da telemedicina, da prescrição nutricional digital e das petições online, a montagem dos planos de treinamento e a quantificação da carga de trabalho serão, cada vez em maior escala, realizadas com maior precisão e rapidez por aplicativos para smartphones (Zhu \& Zheng, 2018).

Essa tendência já se observa também no acompanhamento e controle do treinamento físico. A incorporação de sensores de acelerometria nos telefones celulares e em smartwatches, associada à disponibilidade freeware de aplicativos para estes instrumentos, fazem com que o controle do trabalho realizado, do gasto calórico, e do débito energético já seja realizado prioritariamente por estas tecnologias, ao invés de calculados por um profissional.

\section{CONCLUSÃO}

Como disse o médico Willian Pollard (1911 - 1989): - “Mudar é difícil, não mudar é fatal!”. É natural do ser humano se apegar aos seus hábitos, costumes e paradigmas, porém as drásticas mudanças que nossa sociedade está vivenciando exigem, mais do que nunca, uma adaptação rápida e drástica.

A sociedade 5.0 é a resposta da humanidade para se alcançar os Objetivos de Desenvolvimento Sustentável do Milênio. Estas duas iniciativas potencialmente transformarão o mundo em que vivemos, assegurando um futuro mais justo, sustentável e viável.

No entanto, "não se faz uma omelete, sem quebrar alguns ovos". O atendimento das demandas de forma individualizada, a automação do trabalho, o e-learning, bem como as tendências do mercado de trabalho que foram apresentadas ao longo deste artigo, provocarão profundas transformações nas formas de atuação laboral.

Para tanto, as relações sociais, o trabalho e o modus vivendi de todos nós serão profunda e irreversivelmente afetados por esta "revolução tecnológica", como pode ser visto na Figura 7. 
Figura 7: Os Objetivos de Desenvolvimento Sustentável do Milênio e a Sociedade 5.0

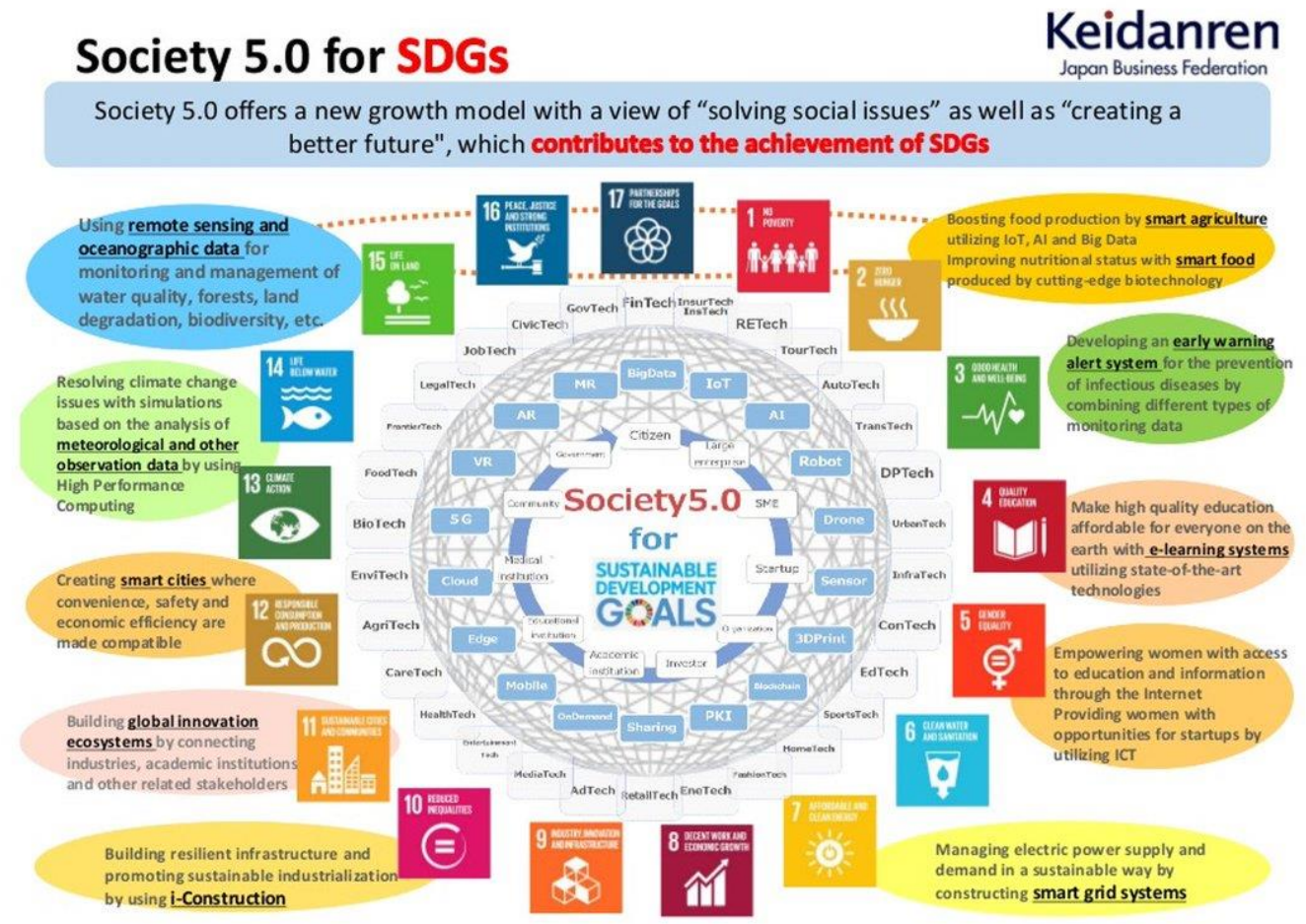

Fonte: Tetsu M., 2019.

Seguindo o conselho de Richard Buckminster Fuller (1895 - 1983):

"Estamos diante de uma relação inteiramente nova com o Universo. Vamos ter que abrir as asas do nosso intelecto e voar, do contrário pereceremos; isto é, devemos voar, imediatamente, por meio dos princípios generalizados que regem o Universo, e não pelas regras rasteiras dos reflexos supersticiosos e erroneamente condicionados do passado" (Fuller, 2001).

Abracemos então, com entusiasmo e determinação, estes novos paradigmas. 


\section{REFERÊNCIAS}

Adebayo, A.O., Chaubey, M. S., Numbu, L.P (2019). Industry 4.0: The Fourth Industrial Revolution And How It Relates To The Application Of Internet Of Things(IoT). Journal of Multidisciplinary Engineering Science Studies (JMESS), Vol. 5 Issue 2.

Azevedo, N. H., \& Santos, S. A. (2017). Telepresence: reflections and basement in environmental law of labor. Nova Hileia, Vol. 2. No 3 .

Alves J.E.D (2019). A revisão 2019 da ONU para as projeções populacionais do Brasil. EcoDebate, ISSN 2446-9394, 21/06/2019. Disponível em https://www.ecodebate.com.br/2019/06/21/a-revisao2019-da-onu-para-as-projecoes-populacionais-do-brasil-artigo-de-jose-eustaquio-diniz-alves/

Bersin, J. (2016). The Future Of Work: It's Already Here - And Not As Scary. Forbes, 187 - 192.

Borba-Pinheiro, C. J., Dantas, E. H., \& Figueiredo, N. M. (2016). Ações Multiprofissionais Sobre o Idoso com osteoporose: Um Enfoque no Exercício Físico. São Caetano do Sul: Yedis.

Cunha, N. C., Cunha, T. N., Costa, S. R., \& Cunha, N. B. (2017). Gestão do conhecimento nas relações da sociedade da informação pela web. Revista Sustinere, 5(1), 114-134.

Dantas, E. H., \& Vale, R. G. (2008). Atividade Física e Envelhecimento Saudável. Rio de Janeiro: Shape.

Foresti, R., Rossi, S., Magnani, M., Lo Blanco, C. G., Delmonte, N. (2020). Smart Society and Artificial Intelligence: Big Data Scheduling and the Global Standard Method Applied to Smart Maintenance. Engineering. v,6, n.7, p. 835-846

Fuller, R. B. (2001). Anthology for the new millennium. Londres: Macmillan Publisher.

Garcia-Muiña, F., González-Sánchez, R., \& Ferrari, A. (2018). The Paradigms of Industry 4.0 and Circular Economy as Enabling Drivers for the Competitiveness of Businesses and Territories: The Case of an Italian Ceramic Tiles Manufacturing Company. Social Science, 7.12: 255- 261.

Hnát, J., Herčko, J., \& Gregor, M. (2018). DESIGN And Control Of Logistic Processes Based On Industry 4.0 Principles. MM science journal, 93 - 99.

Maddox, T. M., Rumsfeld, J. S., \& Payne, P. R. (2018). Questions for Artificial Intelligence in Health Care. JAMA - Journal of American Medical Association, 2018(10), pp. 93 - 96.

Man, D. (2018). Society 5.0":The Way of Implementation of Japan's Super Smart Society. Contemporay Economy of Japan, $03-07$.

4 Maeda, T. Revolução silenciosa: bem-vindos à Sociedade 5.0. Disponível em https://tattimaeda.com.br/revolucao-silenciosa-bem-vindos-a-sociedade-5-0/, Acessado $<03$ de dezembro 2020>

Martynov, V.V., Shavaleeva, D.N., Zaytseva, A.A. (2019). Information Technology as the Basis for Transformation into a Digital Society and Industry 5.0. International Conference "Quality Management, Transport and Information Security, Information Technologies" (IT\&QM\&IS). 23-27 Sept. 2019. DOI: 10.1109/ITQMIS.2019.8928305 
ONU, O. d. (2015, Outubro 15). Transformando Nosso Mundo: A Agenda 2030 para o Desenvolvimento Sustentável. Retrieved Julho 12, 2019, from Organização das Nações Uidas - ONU: https://nacoesunidas.org/pos2015/agenda2030/

Sachs, J. D. (2018). A era do desenvolvimento sustentável. São Paulo: Actual Editora.

Santos, A. B., Veloso, S. L., \& Oliveira, H. A. (2017). A Modernização Da Agricultura E Os Impactos Ambientais: da primeira revolução agrícola dos tempos modernos até os dias atuais. Anais do Simpósio Nacional de Ciência e Meio Ambiente - SNCMA (pp. 2015 -221). Anápolis: Editora do Centro Universitário de Anápolis - UniEVANGÉLICA.

Serviço Nacional da Industria - SENAI. (2018, Julho 06). SENAI aponta 30 novas profissões que vão surgir com a Indústria 4.0. Retrieved Julho 12, 2019, from Serviço Nacional da Industria - SENAI: https://www.fiern.org.br/conheca-30-novas-profissoes-que-vao-surgir-com-industria-4-0/ Shiroishi, Y., Uchiyama, K., \& Suzuki, N. (2018). Society 5.0: For Human Security and Well-Being. CYBER-PHYSICAL SYSTEMS, $91-95$.

Sachsenmeier. P. (2016). Industry 5.0-The Relevance and Implications of Bionics and Synthetic Biology. Engineering. v.2, n. 2, 1, p. 225-229.

Thompson, W. (2018). WORLDWIDE SURVEY OF FITNESS TRENDS FOR 2019. ACSM's Health \& Fitness Journal, 22(6), pp. 10 - 17.

Tidd, J., \& Bessant, J. R. (2018). Managing innovation: integrating technological, market and organizational change. Nova Jersey: John Wiley \& Sons.

Tofler, A. (1973). O Choque do Futuro. Rio de Janeiro: Artenova.

Tetsu, M., G20 Osaka Summit: Progress in "Digital Economy" and "Society 5.0".(2019). Discuss Japan-Japan Foreign Policy Forum, n. 53

United Nations Educational, Scientific and Cultural Organization - UNESCO (2015). UNESCO Science report: towards 2030. Place de Fontenoy. France Paris, 75352. ISBN 978-92-3-100129-1

Vale, R. G., Pernambuco, C. S., \& Dantas, E. H. (2016). Manual de Avaliação do Idoso. São Paulo: Ícone.

Zhu, L., \& Zheng, W. J. (2018). Informatics, Data Science, and Artificial Intelligence. Journal of American Medical Association, 320(11), pp. 1103-1104. . 